The Relationship between Handedness and Valence: A Gesture Study

Esra Nur Çatak ${ }^{1}$, Alper Açık ${ }^{2}, \&$ Tilbe Göksun ${ }^{1}$

${ }^{1}$ Koç University ${ }^{2}$ Özyeğin University

Address for correspondence:

Tilbe Göksun

Department of Psychology, Koç University

Rumelifeneri Yolu, Sariyer 34450

Istanbul - Turkey

Email: tgoksun@ku.edu.tr

Phone: +90-212-338-1872

Fax: +90-212-338-1415 


\begin{abstract}
People with different hand preferences assign positive and negative emotions to different sides of their bodies and produce co-speech gestures with their dominant hand when the content is positive (Casasanto \& Jasmin, 2010). In this study, we investigated this side preference by handedness in both gesture comprehension and production. Participants watched faceless gesture videos with negative and positive content on eye-tracker and asked to retell the stories after each video. Results indicated no difference in looking preferences regarding being right- or left-handed. Yet, an effect of emotional valence was observed. Participants spent more time looking to the right (actor's left) when the information was positive and to the left (actor's right) when the information was negative. Participants' retelling of stories revealed a handedness effect only for different types of gestures (representational vs. beat). Individuals used their dominant hands for beat gestures. For representational gestures while the right-handers used their right hands more, the left-handers gestured using both hands equally. Overall, the lack of significant difference between handedness and emotional content in both comprehension and production levels suggests that the body specific mental representations may not extend to the conversational level. Keywords: handedness, body-specificity, gesture comprehension, gesture production
\end{abstract}




\section{The Relationship between Handedness and Valence: A Gesture Study}

People all have unique, different bodies through which they experience the world.

With this difference in experience, variations in the representations of the world may appear. As these differences in interaction with physical environment are systematic and habitual, their effects on the representations of the owners become more predictable and stable (Casasanto, 2014).

The difference between right-handed and left-handed people offers a way to investigate this body-specificity hypothesis. Hands are the most frequently used parts of the human body to interact with the environment. Right-handed and left-handed people vary in the ability to use both of their hands with the same effectiveness. This difference in dexterity will affect the comfort and the experience in the left or right side of the space they are operating in. According to the body-specificity theory (Casasanto, 2009), because left-handed and right-handed people interact with the world differently, their representations of the world may also vary. In line with this theory, the present study aims to investigate how people's hand preferences influence their comprehension of others' hand gestures that accompany different emotional contents and how they express them in their own gestures. We ask whether (1) right- vs. left-handed people focus on one side of their bodies more than the other, and (2) the extraction of information in gestures differ with the emotional content of stories.

Through many cultures the right side of the space is often paired with goodness, while left side is associated with badness. Many languages are full of different sayings such as "right hand man" (for important people) or "having two left feet" (for clumsy people). For example, in Turkish, the language of this study, the word right "săg" means alive, healthy, and well, while left "sol” means spoilt, broken, and to decay (Nişanyan Dictionary, n.d.). Is this pairing of valence with sides a universally established pattern that is similar for everyone or could this be a result of right-handed people being the majority of the world's population? 
To explain the mechanisms behind this association of valence with space -whether it is a body-specific mapping or simply a cultural phenomenon- a series of experiments were conducted. For example, when English-speaking participants were given two animals to place in the boxes around a character-Bob-, they placed the animal they liked to the left or right of the character according to their own hand preferences. This procedure was called the bob task (Casasanto, 2009). In particular, left-handers placed the "good" animal to the left side and "bad" animal to the right side of the cartoon character most of the time, while right-handers did the opposite. However, when participants were asked to place the animal either above or below the character, the hand preference did not matter. Participants preferred to place the animal they liked in the box above and the other animal in the box below. The study was later replicated in a different culture with Dutch participants to see if people were aware of their choices of placement. Using the same procedure and questions, the results of this follow-up study pointed out that $99 \%$ of the participants were not aware of the purpose of the experiment, meaning that people may not be aware of their valence judgments based on handedness. Additionally, participants significantly chose to place the good animal in the box congruent with their dominant side, even without using their hands. Furthermore, using the same method, de la Fuente, Casasanto, Román, and Santiago (2014) tested Moroccan and Spanish right-handed participants. While Moroccan culture is more salient in "right is good" bias compared to Spanish culture, participants of the two countries produced similar patterns without any cross-cultural differences.

Does the body specificity effect only relate to the natural handedness or can it be also established by motor experience? To examine these questions, Casasanto and Chrysikou (2011) tested patients with weakness or paralysis on one side of their body due to unilateral cerebrovascular accident. These patients were asked to complete the bob task to see the effects of long-term changes in motor fluency. The patients who remained right-handed post 
stroke all selected the right box for the "good animal," while patients who turned left-handed post stroke chose the left box for the "good animal," which was not congruent with their premorbid right-handedness. These findings indicate that the association between valence and space is not due to the natural handedness, but may be a result of acting more fluently on one's dominant side. In a follow up experiment, healthy individuals were turned into left- or right-handers by the experimenters to see whether the results could be the consequence of long-term neural organization due to stroke. First, a motor fluency task was completed with a ski glove either on the right or on left hand of the participant. Then, people completed the bob task. Overall, participants tended to put the good animal to the box on the side congruent with the participants' available hand side during the training phase compared to the side of the hand that had been gloved. Thus, changing people's use of hands even with a brief motor task could lead to a change in their mental representations of valence and space.

People seem to associate goodness with their dominant side whether it has a long- or short-term dominance. Do these associations overreach the situations in which people have to use perspective taking? People may be able to take perspectives of others based on observed or expected bodily characteristics. Kominsky and Casasanto (2013) asked participants to complete the bob task (Casasanto, 2009) by changing perspectives. When people shared the perspective with the character, most of the right-handed participants chose to place the good animal to the animal's and their own right. In the opposite perspective condition, however, when the character was presented facing the participants, they successfully took the character's point of view and leaned towards placing the good animal to the characters right, which was their left side. Moreover, the effect was much more robust when they replicated the study with a real human photograph instead of a drawing character. It seems that people not only associate goodness to their own right, but can also consider another person's perspective in judging valence for them. One other question to be answered about the 
perspective-taking task is whether people consider their own bodily characteristics during perspective taking of others'. The experiment with the human photographs was replicated with a modification, the person in the photographs was wearing a sling on either his right or left arm, making him either right- or left-handed functionally. The results revealed that participants indeed took perspective during valence judgments, considering the other person's bodily state and not projecting their own bodily characteristics onto others.

However, when perspective taking is coupled with vicarious motor experience, rather than taking perspectives, people were more affected by the fluency component. Specifically, de la Fuente and colleagues (2015) made right-handed participants watch right-handed actors completing a motor task by wearing a ski glove in one hand. When both actors and observers completed the bob task, their views on the bad and the good sides differed. Actors put the good animal to their short-term fluent side (left) and the bad animal to their ski-gloved side (right), replicating the earlier Casasanto and Chrysikou (2011) finding. In contrast, observers who watched the motor task phase facing the actors did the opposite. They failed to take perspective and returned to their original good-is-right judgment as the vicarious motor experience strengthened their judgment. These results indicate that the judgments on left and right as good or bad could be a result of specifics of both others' and our own bodies.

The body-specificity hypothesis was also investigated using different formats such as reaction time studies and memory performance (Brunye, Gardony, Mahoney \& Taylor, 2012; de la Vega, de Filippis, Lachmair, Dudschig, \& Kaup, 2012; Rodway, Wright \& Hardie, 2003; van Strien \& van Beek, 2000). For example, right-handed people tended to remember events on a difficult map more to the right side if the event was positive and more to the left side if the event was negative. The opposite was true for left-handed people. This effect was more salient as the handedness of the participants got stronger (Brunye, Gardony, Mahoney \& Taylor, 2012). Yet, for the reaction time and emotion discrimination studies the results are 
more complicated. Earlier studies found no handedness effect in discriminating emotional faces presented either left or right side of the screen (van Strien \& van Beek, 2000; Rodway, Wright \& Hardie, 2003). In a recent study, a lexical decision reaction time procedure was used to see the association between handedness and emotional value of the words. When people were explicitly made to reason the valence judgment and side mapping, an interaction between handedness and emotional valence was present. Yet, when no explicit valence-side mapping instruction was given, the valence judgments on their own failed to activate the association between valence and response side (de la Vega, de Filippis, Lachmair, Dudschig, \& Kaup, 2012). Others also showed that right-handed participants were faster to react to positive stimuli with their right hands and negative ones with their left hands. The opposite was true for left-handed participants (Kong, 2013). Thus, even though there are some conflicting results, the literature strongly supports the body-specificity theory on handedness and emotional valence relationship.

The body specificity theory is not only confined to one's own body, but can expand to our social relationships with others and how we view the perspectives of other people around us (e.g., Kominsky \& Casasanto, 2013). One possible way to examine the relation between handedness and judgments is using hand gestures. Gestures are important for face-to-face communication. During a conversation, speakers spontaneously produce hand movements that support certain aspects of the speech content. When these gestures form a close relationship to the content of speech including shapes, actions, events that are mentioned in the speech, they are classified as iconic gestures. Another gesture type related to accompanying speech is deictic gestures that are pointing movements (McNeill, 1992). Along with the iconic and deictic gestures, the present study will also focus on beat gestures that are movements without a meaning and prototypical in terms of movement characteristics. These gestures are usually slow, rapid flicks taking place wherever the hand happens to rest (McNeill, 1992). The 
investigation of both types of gestures is necessary since representational gestures (iconic and deictic) and beat gestures differ in the way they carry information related to the speech.

Speech and gesture form a coherent language system. Various studies investigating the role of gestures during speech agree on the facilitative effect of gestures on speech (Riseborough, 1981; Kelly, Barr, Church, \& Lynch, 1999; Sueyoshi \& Hardison, 2005). For example, the same story was retrieved better when accompanied with gestures even when the noise level in the environment was high (Riseboroug, 1981, Kelly et al. 1999). Furthermore, participants were less accurate in identifying an action when it was presented to them with incongruent speech and gesture (Kelly et al., 2009). These findings indicate that both speech and gesture affect each other interactively. Finally, Driskel and Radtke (2003) demonstrated that gestures not only aid people's comprehension but also their speech production. People were much better in explaining different contents with gestures. In turn, addressees of a conversation were much better in coming up with answers when they were given gesture information with speech.

Although these studies about the speech-gesture interaction are informative, we need to ask how gestures interact with valence and handedness. A study analyzed presidential election speech videos with two right-handed and two left-handed candidates (Kerry, Bush being right-handed; Obama, McCain being left-handed) (Casasanto \& Jasmin, 2010). The analyses revealed that the valence presented in speech was associated differently for left- and right-handed candidates in gesture. Both left-handed candidates tended to use their dominant hands during the speech containing positive valence clauses and non-dominant hands while they were producing negative valence clauses. The opposite pattern was observed in the righthanded candidates. These results noticeably support the body specificity hypothesis and extend it through a probability of communicative purpose. Speakers may provide the listeners how they feel about the content of the speech they produce at the moment with probably 
unintended cues such as communicative gestures. These results indicate that people's body specific representations blend into the inter-personal level. To see if there are effects of this representation on the communication, the other end of the conversation should be taken into account. Whether these emotionally loaded gestures are perceived as the way they are intended is the next question we aimed to answer in the present study.

\section{The Present Study}

The body-specificity hypothesis suggests that left- and right-handers do not have the same vision of the world. The present study investigates how different people comprehend others depending on their own handedness. We aim to see the relationship between valence and handedness using gestures in an experimental setting. We ask two main questions: (1) Do people perceive positive and negative emotional events that are depicted in left vs. right hand differently according to their hand preferences? (2) How will they, in turn, express this information in their gestures? As in line with the body specificity theory, we hypothesize that during a both-handed gesture, right-handed people will be more likely to attend towards the right hand of the speaker during positive narratives and to the left hand during the negative ones. For left-handed people, we expect the reversed pattern while watching both-handed gestures. However, the pattern could be weaker due to the pre-experimental exposure to righthanded people, who comprise the majority of the population. We also asked participants to tell their idea about the handedness of the gesturer to be included in the analyses as a control variable. Finally, we predict that when later asked to reproduce narratives, people will use their dominant hands more if the content is positive and non-dominant hands if the content is negative. We also expect that individuals will produce more representational gestures depending on what they watched during the videos. For example, for videos in which action gestures are used, people can produce more action related representational gestures due to mimicry. 
To measure the gesture comprehension eye tracking method is used. Eye tracking is a valuable method in the sense that the gaze information recorded gives us understanding on the object of attention and insight on how the scene is perceived by the viewer. For gesture production, we recorded each participant's retelling of the videos and coded them for their verbal and gestural information.

Methods

\section{Participants}

A total of 66 students from Koç University volunteered to participate in the study for monetary award. Twenty-six of the participants were excluded from the eye tracking part: 7 of them due to recording problems during eye-tracking, 16 participants failed to fulfill the descriptions given at the beginning of the sessions (e.g., looking only to the neck or one point on the screen not paying attention to the videos for the full session, or not looking at all as they were told that this was a memory task), and finally 2 participants were discarded due to being ambidextrous. However, we kept the gesture production data of the excluded participants, so while the sample for gesture production analyses consisted of 50 participants (a total of 22 left-handed, 33 females (14 left-handed), 17 males (8 left-handed), the final sample for gesture comprehension analyses consisted of 40 participants, (a total of 19 lefthanded, 27 females (13 left-handed), 13 males (6 left-handed), Mage= 22.65, SDage= 2.8, age range: 19-29), who were given an informed-consent stating that they were free to leave whenever they wanted and that their information would be kept undisclosed to others who were not a part of the research project. All participants were Turkish native speakers.

\section{Measures}

Tobii T120 eye tracker with a data-sampling rate of $60 \mathrm{~Hz}$ was used to record participants' eye movements. The Tobii Studio 3.2.1 was used to obtain the gaze data for the gesture videos. The data recorded by the eye tracker was analyzed with an Area of Interest 
(AOI) approach using Tobii Studio. For the AOIs, the screen was divided into two equal right and left halves from the midpoint. In addition to the entire video, the instances where the gestures included both hands were analyzed separately with different AOI groups (see below for the details of the analyses).

In addition to the gaze data from the eye tracker, people were recorded with a camera throughout the session. After watching each video, they were asked to retell the story. Spontaneous gestures during retelling were analyzed later for the hand preference and content. At the end of the procedure participants completed the Edinburgh Handedness Inventory (EHI) (Oldfield, 1971).

\section{Materials and Procedure}

The testing procedure took place in a silent room for approximately 15 minutes for each participant. The participants were required to sit on an armless chair in front of the eye tracker. At the beginning of the sessions, the procedure was clarified to the participants. They were told to watch and listen the content carefully and to be asked to retell the stories as well as they can remember. The excluded participants from the eye tracking analyses were the ones who failed to watch the videos and most of them claimed that they did not watch the videos to focus on information and to remember the content better later. They focused on one point on the screen regardless of the video type and sometimes we were not able to even record their gaze information. The participants completed the 9-point calibration for eye tracking and after calibration they were presented negative and positive videos in a randomized order.

Participants watched a total of 6 videos; half of them were negative and the other half was positive. The positive event narrations included a birthday celebration, a picnic, and a volleyball victory. The negative narrations consisted of a car accident, failing a class, and a quarrel between roommates. The titles of these stories were presented to the participants before each video to initiate the intended emotions from the start. Additional 23 native 
Turkish university students rated the valence of the narration texts prior to the experiment. These independent raters saw the texts of 8 stories in two different randomized orders and rated the stories between -3 (extremely negative) and 3 (extremely positive) with 0 meaning neutral. The final 6 stories were selected based on the agreement among the participants. Stories rated above and below 1.5 averages were included. Two other stories, which were about $a$ wedding and a sickness, were excluded due to being underrated for the intended emotion.

All videos were recorded with the same female person as the gesturer and narrator without any acting expertise. She wore dark clothing to contrast the hands and her face was not included in the screen. The attire of the narrator was kept gender-neutral. The recording took place in front of a white wall with no other distractions (see Figure 1). The narrator made a total of 6 gestures for each video including 3 types of hand use ( 2 for each): only left, only right, and both hands. The narration was recorded simultaneously with the gestures to ensure the proper mapping of speech to the gestures. The gestures including both hands were separately analyzed for gaze information.

The length of the videos was kept similar to each other to last between 20-30 seconds for each narrative. Figure 1 displays the use of different gestures (object and action) for 3 types of hand use in different scenarios (see below for the use of gestures in the scenario). For the same story texts, speech was accompanied by $\mathbf{3}$ types of gesture conditions: object gestures (static gestures referring to objects), action gestures (dynamic gestures referring to actions) or without gestures as no gesture condition. No specific hypothesis was formulated for action vs. object gestures. We used two different versions to cover the possible natural gestures that could be produced during talking about these emotional events. 
Insert Figure 1 about here

For example, in the same story text for the "Car accident" scenario, in all videos for object, action, and no gesture, the participant heard:

"Bu günlerde çok şanssızım. Daha yeni kar yağdığı için yollar buz tutmuştu. Biz de dün yolda giderken (Figure 1F) kaza yaptık. Kar lastiğini (Figure 1A) henüz takmadığım için araba kaydı (Figure 1D). Trafik lambasına (Figure 1B) geldiğimizde önümüzdeki arabaya çarptık (Figure 1E). Arabanın kaportası tamamen çöktü farlar kırıldı. Keşke hava yastığı (Figure 1C) patlasayd1, biz de bu kadar ciddi yaralanmazdı." (English: "I am so unlucky lately. Because it snowed recently, the roads were icy. So we had a car crash while driving (Figure $1 \mathrm{~F}$ ) on the road yesterday. I did not install the snow tire (Figure 1A) yet, the car slid (Figure 1D). At the traffic lights (Figure 1B) we crashed (Figure 1E) the car in front of us. The hood of the car dented in and our headlights broke. I wish the airbags (Figure 1C) had deployed so we would not be as seriously wounded as we were.") (See Appendix A for the Scripts of all stories)

For different conditions the participants saw different gestures in the same sentence. For example, for the sentence "Dün yolda giderken kaza yaptık" ('We had a car crash while driving on the road yesterday'), the participant saw a gesture accompanying "yolda" ('on the road' gesture: both hand fingers pointing forward move to sides and down) in the object condition and for the action condition they saw a gesture accompanying "giderken" ( while driving' gesture: both hands moving forward with index and middle fingers pointing down and alternate extending). Additionally, there was a no gesture condition without any gestures as a control. All participants viewed six videos in total; two for each type of gesture condition in a randomized order (object gestures, action gestures, and no gesture). In each video, every 
participant watched 6 gestures performed; two gestures for different hand use (left hand only, right hand only, and both hands) (see Table 1 for a sample video presentation to one participant).

Insert Table 1 about here

At the beginning of the procedure, as a cover story, the participants were told that this was a memory study and asked to watch the video carefully to later answer some questions about the narratives. After each video, participants were asked to retell the story in their own words while their speech and spontaneous gestures were recorded. They were not aware of the true purpose of the recording. Finally, participants completed the handedness inventory to assess their level of handedness at the end of the session with a demographic form. They were also asked to tell their own idea about the handedness of the gesturer to be included in the analyses as a perspective taking variable.

\section{Data Coding}

Eye-gaze data. For the whole direction of gaze information, we divided the screen into two Areas of Interests (AOIs), having two identical halves. The AOIs for left and right started when the narration began and lasted until the end of the videos. Later, we exported the visit duration data for the whole video AOIs. For each video, we calculated the percentage of visit duration to the left and right sides. The time participants spent fixating on the AOIs was calculated using Tobii Studio analyze tool. After the AOIs were established, we measured the duration of all visits within an active AOI. An individual visit was defined as the time interval between the first fixation on the active AOI and the end of the last fixation within the same active AOI where there have been no fixations outside the AOI. Since our active AOIs covered the whole screen from left and right, the total visit duration was equal to the duration 
of the videos. We calculated the percentage of the visit duration of only left or right over the total video duration and only conducted analyses on the left side (as left and right sides added up to a hundred).

For the gestures including both hands, first the AOIs were drawn to left and right sides from the exact start of the gestures to 1 second after gesture finished. Participants were slow in reacting to hands and they kept paying attention to one of them after the both-hand gesture finished until another gesture starts. Thus, one second after the end of the both-hand gestures was also included in AOIs. Then, the visit duration for the left and right AOIs were calculated. The analyses were again conducted only for the left side.

Story retelling data. Three independent coders watched the randomly shared recordings of participants' hand movements. The coders transcribed participants' speech, the number of gestures with timestamps, the type of those gestures (beat or representational and static or dynamic), and the meaning of the gesture if it is labelled representational.

From the speech data, we coded how accurate participants recalled the content of the story. We looked for the information supported by gestures in each story in participants' own speech and if half of information in the stories reinforced by gestures was expressed (from 3 out of 6 gestures) the session regarded as accurate. Since the accuracy of the data was only coded for the control purposes, a dichotomous coding was used to eliminate the participants that did not pay enough attention to the task. For example, in the car crash story for the iconic gesture condition if the participant had mentioned at least 3 out of 6 information given verbally and also emphasized in gestures (snow tire, traffic lights, airbag), the session for the video would have coded as accurate.

Spontaneous gestures were coded for the hand use during gesturing. Again, the righthanded and left-handed groups were compared to each other in valence and control conditions for speech and spontaneous gesture data. Gestures of each participant were coded as iconic 
(static, dynamic), pointing or beat with the hand preference information. A gesture was coded as static if it describes an object such as a fist as a ball, while a dynamic gesture was coded if a mobile action was described such as moving one hand forward to indicate running. A pointing gesture was coded when the participants pointed with a finger or whole hand toward the objects around like their own leg or arm. Finally, we coded repetitive meaningless hand movements as beats. Later, for positive and negative stories, gestures used by the left, right or both hands were counted. Gestures performed with both hands were not included in the analyses, as they did not provide us any information other than the participants' general gesturing tendency. The remaining one-hand gestures were analyzed for the hypotheses. Gestures for negative and positive stories were analyzed separately.

\section{Reliability}

The spontaneous gestures were coded by three independent coders and to establish reliability a third person coded the whole sessions of 15 out of 50 participants (30\%); 5 videos from each independent coder. The agreement for the total number of gestures present was $97 \%$ between coders. The agreement between coders for gesture to be classified as beat was $90 \%$ and for representative gesture classification (iconic or pointing) coders agreed $93 \%$.

\section{Control Experiment: Mirror condition}

Following up the experiment, a control condition was run to assess for the possible effects of the gesturer's real-life handedness, and for the possible differences among onehanded gestures. The gesturer in the videos was right-handed. To eliminate the effect of her dexterity difference between sides to the gestures she made, we mirrored the videos horizontally and repeated the earlier experiment with an additional group of right-handed participants.

An additional 24 right-handed participants were volunteered to join the second part of the study for monetary reward. Due to problems in data collection 5 of them were excluded, 
leaving 19 right-handed participants (12 females; Mage=22.37, SDage=2.3, age range: 19 28). The participants were given an informed consent stating that they are free to leave whenever they wanted and that their information will be kept undisclosed to others who are not a part of the research project. All participants were native Turkish speakers. The procedure was the same except the videos were mirrored but shown in the exact order.

$$
\text { Results }
$$

\section{Performed analyses}

For gesture comprehension, we hypothesized that the visit duration to left or right side of the screen would change according to the handedness of the participant and according to the valence of the videos. We performed three-way mixed ANOVA with valence as the within-subjects factor and handedness as the between-subjects factor. We also included perspective taking as a control factor. The analyses were repeated for visit duration for the entire videos and for both hand gestures. Also, the analyses on same factors were repeated comparing mirror condition and original videos.

For production analyses, we used chi-square on gesture frequencies for different conditions and gesture types. We expected an effect of handedness and emotional valence. Therefore, we first compared the use of right and left hand in different handedness groups. Then, we repeated the analyses, separately for beat and representational gestures. Finally, for emotional valence, we compared gesture use (beat and representational gestures together) in positive and negative stories between left- and right-handed participants using chi-square.

\section{Gesture comprehension}

\section{Visit duration: Entire videos}

To examine the total gaze duration for the right and left AOIs of videos with gestures, a three-way mixed ANOVA with emotional valence (negative vs positive) as the withinsubjects factor and participants' own handedness (left vs. right) and perspective taking of the 
gesturer (left-handed or right handed) as the between-subjects factors was performed. The results yielded neither significant main effects (Valence: $F(1,36)=3.34, p=.08, \eta 2=.09$, Handedness: $F(1,36)=.05, p=.83, \eta 2=.001$, Perspective taking: $F(1,36)=.01, p=.92, \eta 2=$ .001) nor any interactions among the variables (between handedness and valence: $F(1,36)=$ $.01, p=.92, \eta 2=.001$, between handedness and perspective taking: $F(1,36)=2.28, p=$ $.14, \eta 2=.06$, between valence and perspective taking: $F(1,36)=.32, p=.57, \eta 2=.01$, among all three variables: $F(1,36)=.20, p=.66, \eta 2=.01)$. Additionally, no video order effect was significant between 3 randomized conditions $(F(1,37)=.80, p=.46, \eta 2=.04)$ and no gender effect was found on the visit duration to the sides $(F(1,38)=.21, p=.65, \eta 2=.01)$. Visit duration: Gesture vs. no gesture videos

For the effect of the presence of gestures, we compared the no gesture videos with their same affect gesture counterparts (positive gesture videos with positive no gesture videos and the same for negatives). For positive videos, a two-way mixed ANOVA with the type of videos (gesture vs no gesture) as the within subject factor and participants' own handedness (left vs. right) as the between-subject factors on visit duration as the dependent variable revealed a main effect of gesture presence $(F(1,38)=5.81, p=.02, \eta 2=.13)$. Without any group difference in handedness $(F(1,38)=1.35, p=.25, \eta 2=.03)$ all participants spent more time looking at the right side of the screen when there were gestures (the viewer's right), compared to the no gesture videos.

For negative videos, we repeated the same analyses and found that there was a significant main effect of the presence of gesture in visit duration to the AOI sides, $(F(1,38)=$ 4.937, $p=.032, \eta 2=.115)$. Compared to the no gesture videos, all participants spent more time looking at the left side of the screen when gestures were present without any group difference in handedness $(F(1,38)=3.34, p=.08, \eta 2=.09)$. 
For visit duration on only both-hand gestures, a three-way mixed ANOVA using emotional valence (negative vs positive) as the within-subjects factor and participants' own handedness (left vs. right) and perspective taking of the gesturer (left-handed or right handed) as the between-subject factors showed a significant main effect of valence, $(F(1,36)=$ 4.299, $p=.045, \eta 2=.107)$. As shown in Figure 2, people spent more time looking at the left side (viewer's left side) while watching negative both-handed gestures than when the video content was positive. No main effect for participant handedness $(F(1,36)=.004, p=.95, \eta 2=$ $.00)$ or the perspective taking $(F(1,36)=1.44, p=.24, \eta 2=.039)$ or any interactions among the variables were obtained (between handedness and valence: $F(1,36)=.04, p=.95, \eta 2=$ .00 , between handedness and perspective taking: $F(1,36)=3.06, p=.08, \eta 2=.08$, between valence and perspective taking: $F(1,36)=1.44, p=.24, \eta 2=.04$, among all three variables: $F(1,36)=.004, p=.95, \eta 2=.00)$.

Insert Figure 2 about here 
We compared the AOI visit duration data from the right-handed participants of the previous analyses and a new group of right-handed participants who watched the mirrored version of the same videos. A mixed ANOVA results indicated no effect of condition (mirror vs. original) on the left visit duration for the whole video and also for only both-handed gesture AOIs (for whole videos: $F(1,37)=1.477, p=.241, \eta 2=.074$, for only both hand gestures: $F(1,37)=.009, p=.991, \eta 2=.000)$. The actor handedness idea by condition was, however, significant, $(\chi 2(1, \mathrm{~N}=40)=6.81, \mathrm{p}=.012)$. While in the original condition only $38.1 \%$ of the participants thought that the gesturer was left-handed, in the mirror condition $78.9 \%$ of the participants thought the gesturer was left-handed.

\section{Gesture production}

Speech: Accuracy

For speech, overall accuracy for retelling the stories was $64 \%$, and accuracy information from speech had no effect on our results, $(\chi 2(1, N=1809)=.221, p=.645)$. That is, participants' correct recall of information presented in the videos did not influence total gesture production by handedness. All participants have given accurate descriptions to some extend and none of the accounts or the whole information from the participants was excluded. Thus, we did not account for accuracy in the following analyses.

\section{Gestures: Both-handed vs. one-handed}

A total of 1208 gestures from 52 participants were obtained. Four participants ( 1 lefthanded and 3 right-handed) produced no gestures through the session and were excluded from these analyses. There was no significant difference in producing both-handed (44\%) vs. onehanded gestures $(56 \%),\left(\chi^{2}(1, N=1208)=1.44, p=.23\right)$.

\section{Gestures: Left-handed vs right-handed}

The percentage of left- and right-hand gestures differed by handedness; both groups preferred to use their dominant hands for one-handed gestures, $(\chi 2(1, N=688)=56.02, p=$ 
.001). The left-handed participants used their left hand for $63 \%$ of the one-handed gestures whereas right-handed participants used their right hand for $64 \%$ of their gestures regardless of emotional valence.

\section{Gestures: Representational and beat}

For a more detailed analysis, we analyzed beat and representational gestures (iconic and pointing together) separately for hand use by handedness groups. A total of 535 onehanded beat gestures were produced during retelling task: 246 of these gestures came from the left-handed participants and 289 from the right-handed participants. The percentage of the right- vs. left-handed beat gestures differed by handedness $(\chi 2(1, N=535)=66.64, p=.001)$. The left-handed participants used their left hands for $72 \%$ of the beat gestures while the righthanded participants preferred to use their right hand for $63 \%$ of the one-handed beat gestures.

We coded a total of 158 one-handed representational gestures (49 by the left-handed participants and 109 by the right-handed participants). The use of left vs. right hand by group was significantly different $(\chi 2(1, N=158)=4.65, p=.001)$. For the right-handed group, $74 \%$ of the representational gestures was produced by the dominant right hand, however there was no hand preference in the left-handed group, almost half (43\%) of the representational gestures were produced by left hand.

\section{Gestures: Dynamic vs. static}

We compared the number of dynamic and static gestures produced by the participants after watching action and object videos. The results indicated that there was an effect of video type over the gesture types produced $\left(\chi^{2}(1, N=177)=7.13, p=.01\right)$. After watching the action videos, $57 \%$ of the gestures produced were dynamic gestures, while after participants watched an object video $63 \%$ of the gestures they produced were classified as static gestures indicating a mimicking effect. 


\section{Gestures: Emotional valence}

For emotional valence, we compared gesture use (beat and representational gestures together) in positive and negative stories between left- and right-handed participants. The analyses revealed no effect of emotional valence on the number of gestures produced with left or right hand for either left- or right-handed groups (for left-handed group: $\chi^{2}(1, N=309)=$ $.95, p=.33$, for right-handed group: $\left.\chi^{2}(1, N=379)=.06, p=.80\right)$. The same results were obtained when the analyses were repeated separately for beat and representational gestures for each handedness group, (for representational gestures: left-handed group: $\chi^{2}(1, N=49)=$ $1.87 p=.17$, right-handed group: $\chi^{2}(1, N=109)=.06, p=.81$; for beat gestures: left-handed group: $\chi^{2}(1, N=246)=.01, p=.94$, for right-handed group: $\left.\chi^{2}(1, N=289)=.03, p=.87\right)$.

\section{Discussion}

The body specificity hypothesis claims that the differences between people's bodies affect their mental representations (Casasanto, 2009). More specifically, people with different hand preferences form emotional mental representations for their dominant hand-side as a product of experiencing better fluency. In this study, we investigated whether individuals' handedness had an effect on perceiving positive and negative emotional events that were depicted in gestures. We also asked whether people would in turn express similar information in their gestures. Based on the findings from the earlier studies on the body specificity theory, we hypothesized that (1) there would be a difference in the way people recruit information during a conversation according to their hand preference and the emotional value of the videos, and (2) people would differ in reproducing this information in gestures according to their handedness and the emotional valence.

Our results indicated no difference for the hand preference. The left-handed and righthanded people displayed no dissimilarity of preference to the left or right side visit duration for positive or negative emotional valence videos. However, we found an effect of emotional 
valence on the visit duration to sides regardless of handedness, in which right and left space were associated with positive and negative valence, respectively. Lastly, for the gesture production, we found that right- and left-handed groups had differences in using their hands for gestures and this effect depended on gesture type (beat vs. representational).

\section{Valence and Handedness}

Studies on handedness and emotional valence show that people link positive ideas with their dominant hand side (Casasanto, 2009; Casasanto \& Chrysikou, 2011; Fuente et al., 2014; de la Fuente et al., 2015). Moreover, in a conversation people hint these representations through their gestures, using their dominant hands while talking about positive topics and non-dominant hands during negative conversations (Casasanto \& Jasmin, 2010). Since it takes two to communicate, we wanted to understand if the conversation partners were able to take these body specific hints.

Throughout our stimuli videos or the instances of both-hand gestures in each video, there was no difference in looking at the left or right side of the screen between handedness groups. Only for both-handed gestures, emotional valence of the stories affected participants' information collection. When the stories were positive, people preferred to look at the right side of the screen (the actor's left side) and when the stories were negative, the preference leaned towards the left (the actor's right side). There was no significant effect for people's own hand preference or people's ideas on the actor's hand preference, indicating that the perspective of the actor was not taken into account. Nevertheless, the number of participants who thought the actor was left-handed was small compared to the rest of the participants who thought the actor was right-handed, these results may not be completely conclusive.

Furthermore, for both the mirror and original videos we found no effect of gaze information by handedness or valence, yet most of the participants in the mirror condition still thought that the gesturer was left-handed. An explanation for this finding could be the 
salience difference between gestures; our gestures were not the same for left and right hands. Some of these gestures might be more memorable for the participants, causing a deduction for the gesturer's handedness at the end of the session. Nevertheless, the lack of difference in gaze duration for sides for both-handed gestures shows that the real-life handedness of the gesturer had no effect on our results.

Still, there is some evidence indicating the pairing of "good" with right side of space and "bad" with the left side could be beyond practical handedness. One reason could be a byproduct of a right-handed society, in which $90 \%$ people are right-handed. Thus, individuals have more exposure to right-handed people and as a result even left-handed people may modify their pairing of good and bad with the right and left side of the space. Furthermore, since our study was conducted with Turkish participants, the effect of Turkish culture that supports the "good is right" way of thinking can have an influence on our findings. Yet, the generality of the right-good association across cultures is promising regarding the crosscultural generalizability of the present results.

In another aspect, our results could also be explained by the valence hypothesis, which suggests that the right hemisphere is specialized for negative emotions and the left hemisphere is specialized for positive emotions (Silberman \& Weingartner, 1986). One of the studies confirming this hypothesis was by Rodway, Wright, and Hardie (2003). They reported increased accuracy for emotion recognition on the positive faces appearing on the right-hand side and negative faces on the left-hand side of the screen and no handedness difference among conditions for accuracy. This tendency of linking positive with right and negative with left regardless of handedness was also demonstrated in our study and hemispheric specialization could be a reason for this effect.

Overall, we did not find any evidence for the interaction of handedness and valence in any level of our analyses on gaze information. Our stimuli were complex compared to the 
previous stimuli used in body specificity studies such as the bob task or reaction-time tasks that focuses on one specific bias. One question from our study was whether complex stimuli that have more resemblance to a real-life situation can evoke the body specific biases as well. Our results demonstrate that along with different variables and other biases we employ in real life; our assumed body specific biases may not be very effective. However, given the mixed results on people's eye gaze directions in various stimuli, more research is needed to generalize our findings.

\section{Gesture production}

After watching each video, we asked participants to retell the stories and recoded their gestures to see if the emotional characteristics of the stories would affect the hand they preferred to use while spontaneously gestured. In general, there was no difference in frequency for both-handed and one-handed gestures produced by participants. Yet, when the beat and representational gestures were compared, beat gestures were four times more frequent than the representational gestures. The reason for recoding a larger number of beat gestures could be our cover story as a memory task. Since beat gestures and non-iconic gestures were found to facilitate target word production (Lucero, Zaharchuk \& Casasanto, 2014), the need to remember the words for the task could encourage beat gesture use.

There was no effect documented for emotional valence on the hand choice of gesture. We only found an effect of handedness: the left-handers used their left hands more than their right hands and the exact opposite pattern was observed for right-handers. Our results did not replicate the study by Casasanto \& Jasmin (2010), in which they stated that politicians with different hand preferences used their dominant hand more during positive utterances and nondominant hand more during negative speech. The reason for the difference may lie in the methods and population included. The present study was a controlled experiment with the emotional characteristics of speech accompanying gestures were manipulated whereas 
Casasanto and Jasmin's (2010) study examined previously recorded videotapes as case studies and had naturalistic data regarding emotional valence. We manipulated the emotional valence through regular, everyday stories with generally involving negative or positive connotations. These stories may have different meanings for some of our participants due to preexperimental exposure. For example, if resolved with a positive outcome, one could have a positive memory of a car crash. The same conflict is also present for the politicians included in Casasanto and Jasmin (2010) however; the ratings of positive and negative utterances were done by independent raters while politicians themselves may not agree on the emotional value of the utterances. Future research can include self-ratings on the emotional valence component.

Moreover, our participants listened to the stories knowing that they had to retell it as accurately as possible. This may have distanced them from the emotional value of the stories. In a natural conversation setting, we do not only focus on the emotional content, as there are so many variables and many other biases to consider.

Another phenomenon that could have an effect on our gesture production results could be the co-speech gesture mimicry. In the present study, the participants have been affected by the gesturer's movements in the sense of the type of the gestures and/or the shape of the movements. They produced more dynamic gestures after watching an action gesture video and more static gestures after viewing an object gesture video. However, as the number of left, right or both-hand gestures was equally distributed in all through the videos, the effect of mimicry on the participants' choice of hand in production was kept to minimum.

Small sample size could be a factor for our results. Because this is a handedness study, including data from left handed population, we believe the number of participants included was reasonable. Additionally, the previous studies on the body specificity hypothesis that reported significant difference by handedness included no more than the number in our study, 
if they had included left handed participants at all (see Kong, 2013; Van Strien \& Van Beek, 2000; Casasanto et al., 2015; Willems et al., 2010). Thus, even though our sample size can be low, we believe the results can be comparable to the previous studies.

Finally, our population included university students with a close profile to general layperson, while the politicians included in Casasanto and Jasmin's (2010) study probably had speech and body language trainings and with a purpose of persuading people, which would lead to more awareness and control over one's gestures.

Our gesture production results also indicate that in addition to the frequency, the quality of the gesture production was significantly different for handedness groups. For beat gestures, right- and left-handed participants preferred to use their dominant hands more, similar to the general gesture production results. However, when participants produced representational (iconic and pointing) gestures, only right-handed participants preferred to use their dominant hands more frequently. The left-handed participants did not choose to use one hand more compared to the other. The hand preferences for different types of gestures have been a topic of research and mixed findings were reported. Studies mostly included righthanded participants only and found a right-hand preference for representational gestures (Sousa-Poza, Rohrberg, \& Mercure, 1979; Stephens, 1983; Blender et al., 1995; Foundas et al., 1995). For non-representational gesture use, the findings were diverse. Sousa-Poza, Rohrberg, \& Mercure (1979) and Stephens (1983) reported no difference in hand preference of right-handers for non-representational type of gestures, Blonder and colleagues (1995) reported a left hand, Foundas and colleagues (1995) reported a right-hand preference for right-handed individuals. One of the few studies that included left-handed participants as a group reported that people used their dominant hand for representational gestures and there were no reliable differences in handedness for beat gestures (Stephens, 1983). 
Deriving from a hemispheric specialization of different language tasks hypothesis, Kita, Condappa, and Mohr (2007) compared hands preferred by right-handers for metaphorical, concrete and abstract sentences in spontaneous gesture production tasks. They found a significantly weaker right-hand preference for the metaphor condition compared to the non-metaphorical conditions for iconic gesture use. As right hemisphere is associated with language skills such as metaphor and humor processing, these results suggest that activation of the right hemisphere in metaphor context may reduce the tendency to use the left hemisphere for gesture generation, thus, decreasing the likelihood of right hand use.

The present results for no hand preference in left-handed participants for representational gestures could be explained by the hemispheric symmetries present in lefthanders for language lateralization. Various studies reported asymmetric left hemisphere lateralization for right-handed people and atypical-symmetric lateralization for left-handed people. Representational gestures particularly relate to language. Additionally, representational gestures activate language areas in the brain (Dick et al., 2009). The organization of language activation in hemispheres reflects itself in our representational gesture production as well. The right-handers who employed their left hemisphere for language production preferred to use their right hands more often than their left hands. In contrast, the left-handers who tended to have a symmetrical activation in two hemispheres did not prefer one hand to the other for representational gestures. Thus, our spontaneous gesture production findings during retelling add to the literature about the close relation between speech production and gesture use based on handedness.

\section{Conclusion}

Our study investigated handedness, emotional valence, and gesture associations in a novel format using eye gaze information to present a possible link among these variables. We found no effect of handedness for gesture comprehension of negative and positive scenarios, 
but a general effect of emotional valence was demonstrated for looking at different sides of the screen. In addition, when we asked participants to retell the stories they watched, there was a difference in the preference of using left vs. right hands based on handedness, but only for the representational gestures. Emotional valence did not influence the hand participants used for gestures.

Our findings shed light on to the literature on the body specificity hypothesis, adding evidence from eye gaze direction in gesture comprehension and gesture production in story retelling. Although the findings contradict to the previous literature on the body specificity hypothesis, this may be due to the task difference or experimental design. Alternatively, the body specific mental representations may fail to spread out to the conversational level. 


\section{References}

Blonder, L. X., Burns, A. F., Bowers, D., Moore, R. W., \& Heilman, K. M. (1995). Spontaneous gestures following right hemisphere infarct. Neuropsychologia, 33(2), 203-213.

Brunyé, T. T., Gardony, A., Mahoney, C. R., \& Taylor, H. A. (2012). Body-specific representations of spatial location. Cognition, 123(2), 229-239.

Casasanto, D. (2009). Embodiment of abstract concepts: good and bad in right-and lefthanders. Journal of Experimental Psychology: General, 138(3), 351.

Casasanto, D. (2014). Bodily Relativity. Routledge Handbook of Embodied Cognition. L. Shapiro (Ed.) pp. 108-117. New York: Routledge.

Casasanto, D., \& Chrysikou, E. G. (2011). When left is "right" motor fluency shapes abstract concepts. Psychological Science, 22(4), 419-422.

Casasanto, D., \& Jasmin, K. (2010). Good and bad in the hands of politicians: Spontaneous gestures during positive and negative speech. PLoS One, 5(7), e11805.

de la Fuente, J., Casasanto, D., \& Santiago, J. (2015). Observed actions affect body-specific associations between space and valence. Acta Psychologica, 156, 32-36.

de la Vega, I., De Filippis, M., Lachmair, M., Dudschig, C., \& Kaup, B. (2012). Emotional valence and physical space: Limits of interaction. Journal of experimental psychology: human perception and performance, 38(2), 375.

de la Vega, I., Dudschig, C., De Filippis, M., Lachmair, M., \& Kaup, B. (2013). Keep your hands crossed: The valence-by-left/right interaction is related to hand, not side, in an incongruent hand-response key assignment. Acta Psychologica, 142(2), 273-277.

Dick, A. S., Goldin-Meadow, S., Hasson, U., Skipper, J. I., \& Small, S. L. (2009). Co-speech gestures influence neural activity in brain regions associated with processing semantic information. Human Brain Mapping, 30(11), 3509-3526. 
Dobel, C., Diesendruck, G., \& Bölte, J. (2007). How writing system and age influence spatial representations of actions a developmental, cross-linguistic study. Psychological Science, 18(6), 487-491.

Driskell, J. E., \& Radtke, P. H. (2003). The effect of gesture on speech production and comprehension. Human Factors: The Journal of the Human Factors and Ergonomics Society, 45(3), 445-454.

Foundas, A. L., Macauley, B. L., Raymer, A. M., Maher, L. M., Heilman, K. M., \& Rothi, L. J. G. (1995). Gesture laterality in aphasic and apraxic stroke patients. Brain and Cognition, 29(2), 204-213.

Fuente, J., Casasanto, D., Román, A., \& Santiago, J. (2014). Can Culture Influence BodySpecific Associations Between Space and Valence? Cognitive Science, 39, 821-832.

Holle, H., Obleser, J., Rueschemeyer, S. A., \& Gunter, T. C. (2010). Integration of iconic gestures and speech in left superior temporal areas boosts speech comprehension under adverse listening conditions. Neuroimage, 49(1), 875-884.

Karev, G. B. (1999). Directionality in right, mixed and left handers. Cortex,35(3), 423-431.

Kebbe, H., \& Vinter, A. (2013). How culture, age, and manual dominance affect directionality in drawing side view objects. Journal of Cross-Cultural Psychology, 44(1), 160-172.

Kelly, S. D., Barr, D. J., Church, R. B., \& Lynch, K. (1999). Offering a hand to pragmatic understanding: The role of speech and gesture in comprehension and memory. Journal of Memory and Language, 40(4), 577-592.

Kelly, S. D., Kravitz, C., \& Hopkins, M. (2004). Neural correlates of bimodal speech and gesture comprehension. Brain and Language, 89(1), 253-260.

Kelly, S. D., Özyürek, A., \& Maris, E. (2009). Two Sides of the Same Coin Speech and Gesture Mutually Interact to Enhance Comprehension. Psychological Science. 21(2), 260-267. 
Kita, S., de Condappa, O., \& Mohr, C. (2007). Metaphor explanation attenuates the right-hand preference for depictive co-speech gestures that imitate actions. Brain and Language, 101(3), 185-197.

Kominsky, J. F., \& Casasanto, D. (2013). Specific to whose body? Perspective-taking and the spatial mapping of valence. Frontiers in Psychology, 4.

Kong, F. (2013). Space-valence associations depend on handedness: evidence from a bimanual output task. Psychological research, 77(6), 773-779.

Lucero, C., Zaharchuk, H., \& Casasanto, D. (2014). Beat gestures facilitate speech production. In Proceedings of the 36th Annual Meeting of the Cognitive Science Society (CogSci 2014) (pp. 1694-1699).

McNeill, D. (1985). So you think gestures are nonverbal?. Psychological Review, 92(3), 350.

McNeill, D. (1992). Hand and mind: What gestures reveal about thought. University of Chicago Press. Chicago.

Oldfield, R. C.(1971).The assessment and analysis of handedness: the Edinburgh inventory. Neuropsychologia 9, 97-113.

Riseborough, M. G. (1981). Physiographic gestures as decoding facilitators: Three experiments exploring a neglected facet of communication. Journal of Nonverbal Behavior, 5(3), 172-183.

Rodway, P., Wright, L., \& Hardie, S. (2003). The valence-specific laterality effect in free viewing conditions: The influence of sex, handedness, and response bias. Brain and Cognition, 53(3), 452-463.

Sağ (Right), Sol (Left) (n.d.). In Nişanyan Turkish Etymology Dictionary. Retrieved from http://www.nisanyansozluk.com/?

Silberman, E.K., Weingartner, H., 1986. Hemispheric lateralization of functions related to emotion. Brain and Cognition, 5, 322-353. 
Sousa-Poza, J. F., Rohrberg, R., \& Mercure, A. (1979). Effects of type of information (abstract-concrete) and field dependence on asymmetry of hand movements during speech. Perceptual and Motor Skills, 48(3c), 1323-1330.

Stephens, D. (1983). Hemispheric language dominance and gesture hand preference (Doctoral dissertation, University of Chicago, Department of Behavioral Sciences, Committee on Cognition and Communication).

Sueyoshi, A., \& Hardison, D. M. (2005). The role of gestures and facial cues in second language listening comprehension. Language Learning, 55(4), 661-699.

Van Strien, J. W., \& Van Beek, S. (2000). Ratings of emotion in laterally presented faces: Sex and handedness effects. Brain and Cognition, 44(3), 645-652. 
Acknowledgements

This research was partially supported by BAGEP Award (Turkish Science Academy Young Investigator Award) given to Tilbe Göksun. We would like to thank everyone in the Language and Cognition Lab at Koç University for their helpful comments in this research, Aylin Küntay and Çağla Aydın for their constructive feedback on the findings. We are grateful to Ayşenur Karaduman for her help in stimulus preparation, and Benay Başkurt, Atakan Kaya, Melike Hazır, and Büşra İzgi for their help in data collection and coding. 
Table 1.: Sample combination of videos for one participant

Gesture types Positive Content Negative Content

\begin{tabular}{l|ll}
\hline Object & A birthday celebration & A car accident \\
Action & A volleyball victory & Failing a class \\
No gesture & A picnic & A quarrel
\end{tabular}


Figure Captions

Figure 1. Examples of different hand gestures for object condition, representing snow tire
(A), traffic lights (B), airbags (C) and for action condition representing sliding (D), crashing $(\mathrm{E})$, driving $(\mathrm{F})$.

Figure 2. The mean percentages of visit duration spent in the left side of the screen (actor's right hand side) only for both-handed gestures for positive and negative conditions. ${ }^{*} p<.05$. 


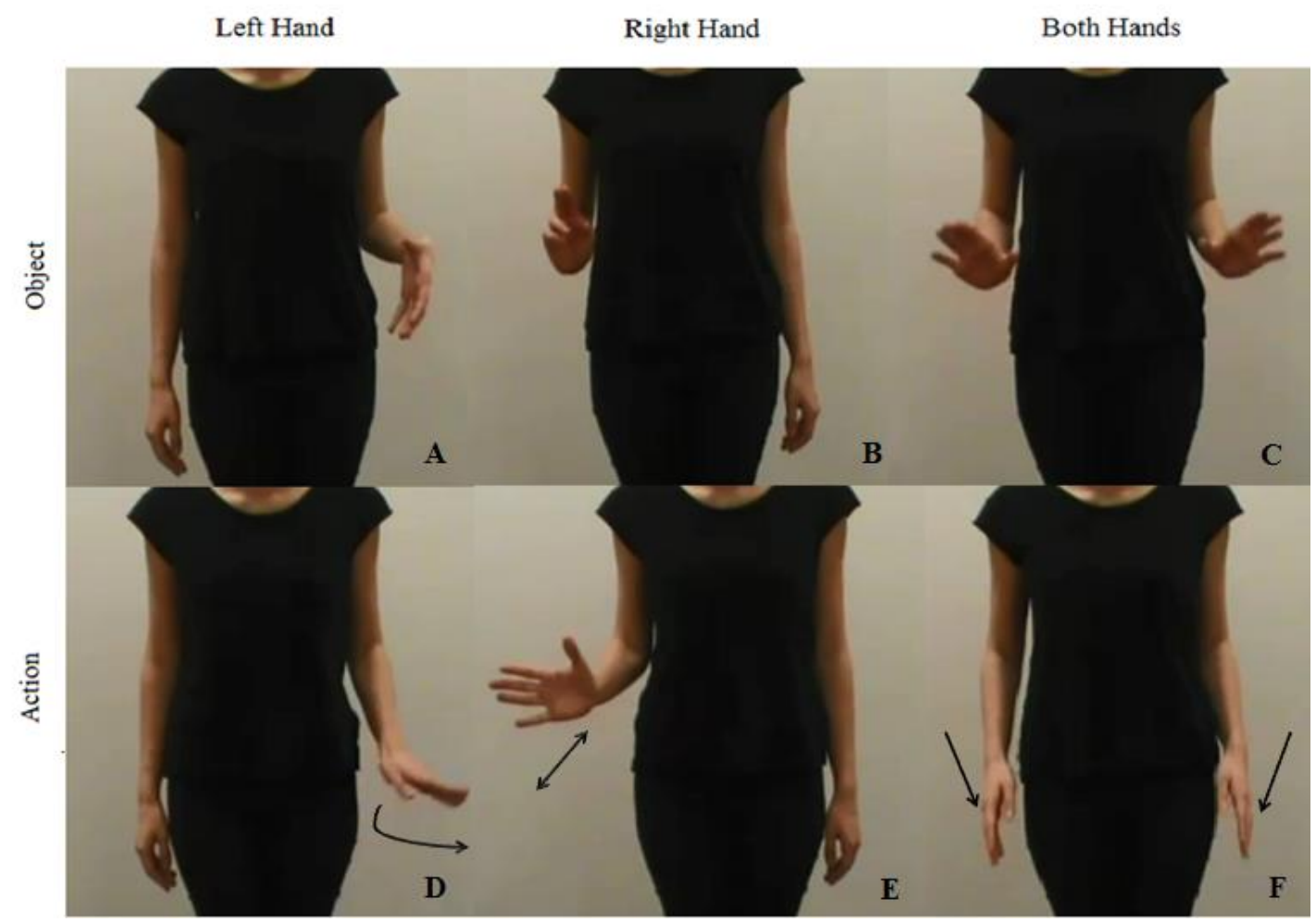

Figure 1. Examples of different hand gestures for object condition, representing snow tire (A), traffic lights (B), airbags (C) and for action condition representing sliding (D), crashing (E), driving $(\mathrm{F})$. 


\section{Both Hand Gestures}

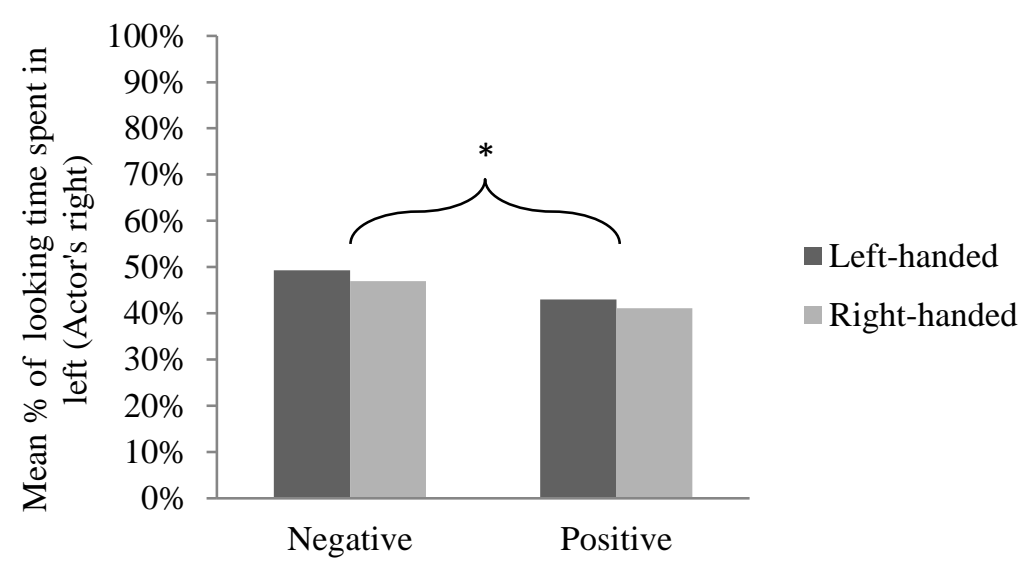

Figure 2. The mean percentages of visit duration spent in the left side of the screen (actor's right hand side) only for both-handed gestures for positive and negative conditions. ${ }^{*} p<.05$. 


\section{Appendix A}

\section{Script Texts for Positive and Negative Stories}

\section{Bold: action gestures}

$\underline{\text { Underlined: object gestures }}$

Positive Scenarios:

\section{Scenario 1: Piknik - Picnic}

Ne zamandır hava çok güzel! Biz de dun ufaklıklarla(sol) pikniğe gittik(sol). Yerleştikten hemen sonra mangalı(iki el) yaktık(să̆). Yemekten sonra biz sohbet ederken(iki el), çocuklar(sağ) da bütün gün etrafta(sol) koşuşturdular(sol). Bir ara birlikte ip atlayıp(să ) top(sağ) oynadık ama en beğendikleri aktivite uçurtma(iki el) uçurmaktı(iki el).

The weather has been very nice lately! So we went on (left) a picnic with the little kids (left). After settling down, we started the fire (right) for the barbecue (both hands). After dinner as we chat (both hands), kids (right) ran (left) around (left) all day. At some time, we jumped rope (right) and played ball (right) together, but their favorite activity was flying (both hands) the kite (both hands).

\section{Scenario 2: Doğum günü - Birthday}

Bugün benim doğum günüm! Arkadaşlarımla pasta(sağ) keserek(sağ) kutladık. Mumları(iki el) üfledikten(iki el) sonra sürpriz hediyelerini(sol) aldığımda(sol) çok heyecanlandım. En büyük hediye kutusunu(iki el) açtığımda(iki el) ne gördüm dersiniz? Hep istediğim yavru köpek(sol) birden dışarı atlayıp koşmaya(sol) başladı. Uzun bir süre evin içinde(sağ) kahkahalarla onu yakalamaya (sağ) uğraştık.

Today is my birthday! We celebrated with friends by cutting (right) a cake (right). After I blew (both hands) the candles (both hands), I got excited when I got (left) their 
surprise presents (left). When I opened (both hands) the biggest box (both hands), what did you think I saw? The puppy (left) I always wanted jumped right up and started running (left). For a long time we chased (right) it around (right) the house with laughter.

Scenario 3: Maç kazanma - Winning a game

Dün ilk voleybol maçımı kazandım! Maçtan önce o kadar heyecanlıydım ki

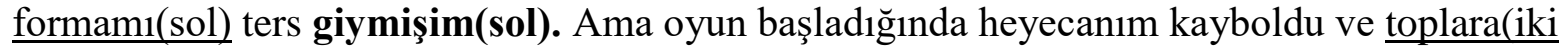
el) düşünmeden koşmaya(iki el) başladım. Oyunun sonunda skor tahtası(sağ) bizim kazandığımızı göstererek yanıp söndü(săg). Ardından bütün seyircilerle(sol) toplanıp sarılarak(sol) zaferimizi kutladık. Çıkışta koçun aldığı pastayı(sağ) kestik(sağ). Bütün gece, şişirdiğimiz balonlarla(iki el) biraz daha voleybol oynayıp(iki el) kutlama yaptık.

I won my first volleyball game! I was so excited before the game I wore (left) my uniform (left) inside out. When the game started, my excitement went away and I ran (both hands) to the balls (both hands) without thinking. At the end of the game the score table (right) flashed (right) indicating our win. After that we celebrated our victory hugging (left) the audience (left). In the end we cut (right) the cake (right) our coach brought for us. And through the night we blew some balloons (both hands) and kept playing (both hands) some more volleyball with them.

Negative Scenarios:

\section{Scenario 1: Araba kazası - Car accident}

Bu günlerde çok şanssızım. Daha yeni kar yağdığı(sağ) için yerler(sağ) buz tuttu. Biz de dün yolda(iki el) giderken(iki el) kaza yaptık. Kar lastiğini(sol) henüz takmadığım için araba kaydı(sol). Trafik ıșıılarına(sağ) geldiğimizde önümüzdeki arabaya çarptık(sağ). Arabanın kaportası tamamen çöktü(sol) farlar(sol) kırıldı. Keşke arabanın hava yastığı(iki el) patlasaydı(iki el), bu kadar ciddi yaralanmazdık. 
I am so unlucky lately. Because it snowed (right) recently the roads (right) were icy. So we had a car crash while driving (both hands) on the road (both hands) yesterday. Because I didn't install the snow tire (left) yet the car slid (left). At the traffic lights (right) we crashed (right) into the car in front of us. The hood of the card dented in (left) and our headlights (left) broke. I wish the airbags (both hands) had deployed (both hands) we wouldn't be as seriously wounded as we are.

\section{Scenario 2: Dersten kalma - Failing classes}

Bu dönem iki dersten kaldım. Final sabahı telefonun(sol) alarmını kapatıp(sol) uyuyakalmışım. Telafi için hocayla konuştuğumda ise ödev(sağ) yazabileceğimi(sağ) söyledi. Ama maalesef ödevi yazmadan önce dışarıda bisikletten(iki el) düştüm(iki el). Bileğim ve dizim(sağ) incindiği için yürüyemiyordum(să̆). İyileşene kadar yatağımda(iki $\underline{\text { el) }}$ yatmak(iki el) zorunda kaldım. Telafiye girebileceğimi söylediler fakat sadece kitap(sol) okuyarak(sol) çalışabildiğim için geçer not alamadım.

I failed two classes this semester. At the finals morning I put of the alarm (left) of my phone (left) and overslept. When I talked to the professor for compensation she told me I could write (right) an assignment (right). But, before finishing it I fell (both hand) from my bike (both hands). My wrist and knee (right) was injured and I couldn't walk (right). I had to lie down (both hands) on my bed (both hands) until I got better. I was told I could attend the make-up test however I could only study by reading (left) the books (left), and so I couldn't get a passing score.

\section{Scenario 3: Tartışma - Quarrel}

Sonunda ev arkadaşlarımla kavga ettim. Öncelikle anahtarları(sağ) olmasına rağmen sürekli zile basıyorlar(să̆). Yemek yedikleri tabakları(iki el) mutfak masasına öylece bırakıyorlar(iki el). Banyonun musluğunu(sol) hiçbir zaman tam olarak kapatmadıkları(sol) için küvete(sağ) boşu boşuna su akıyor(sağ). En sinir bozucu olanı da 
bunları tartışmak(iki el) istediğimde kapıları(iki el) sımsıkı kapatılmış oluyor. Ya da çok aceleleri olduğundan çantalarını(sol) kapıp koşarak(sol) evden çıkıyorlar.

Finally we had a quarrel with my home mates. First of all, although they have the keys (right), they still ring (right) the doorbell. They leave (both hands) the plates (both hands) on the table without cleaning. And since they never turn off (left) the faucet (left), the water keeps running (right) down the tub (right). The most annoying part is that whenever I try to talk (both hands) to them about these things they close the doors (both hands) tightly or they grab their bags (left) and run out (left) because of an emergency. 